\title{
Near-field photonic forces
}

\author{
By M. Nieto-Vesperinas ${ }^{1}$, P. C. Chaumet ${ }^{2}$ and A. Rahmani ${ }^{3}$ \\ ${ }^{1}$ Instituto de Ciencia de Materiales de Madrid, Consejo Superior de \\ Investigaciones Cientificas, Campus de Cantoblanco, \\ Madrid 28049, Spain (mnieto@everest.icmm.csic.es) \\ ${ }^{2}$ Institut Fresnel (UMR 6133), Faculté des Sciences et Techniques \\ de St Jérôme, Av. Escadrille Normandie-Niemen, \\ Marseille 13397 CEDEX 20, France (pchaumet@loe.u-3mrs.fr) \\ ${ }^{3}$ Laboratoire d'Electronique, Optoélectronique et Microsystèmes-UMR 5512, \\ Centre National de la Recherche Scientifique, Ecole Centrale de Lyon 36, \\ Av. Guy de Collongue, BP 163, Ecully 69131 CEDEX, France \\ (adel.rahmani@ec-lyon.fr)
}

Published online 17 February 2004

A review of recent advancements in photonic forces is presented. We discuss in detail the interaction of light and sub-wavelength particles on a substrate illuminated by total internal reflection, and we study the optical forces experienced by the particles. The effects of plasmon-mode excitations on the resulting photonic forces on metallic particles are also addressed. Moreover, we explore the possibility of using the metallic tip of a classical apertureless microscope to create optical tweezers, and thus to achieve a selective manipulation of nanoparticles.

Keywords: photonic forces; optical tweezers; plasmon resonance

\section{Introduction}

The manipulation of micro- and nanostructures by means of the mechanical action of light on matter has opened a new field of study at these scales. In 1986 the optical tweezer was invented (Ashkin et al. 1986) as a by-product resulting from extensive studies of the radiation pressure exerted by light on cells, particles and atoms. Ever since, this device has permitted the non-destructive handling of structures in a variety of techniques ranging from spectroscopy (Sasaki et al. 1991; Misawa et al. 1992), phase transitions in polymers (Hotta et al. 1998) and light-assisted ordering of dielectric particles (Burns et al. 1990) to photonic force spectroscopy of cells (Pralle et al. 1998) and biological molecules (Smith et al. 1996).

The high resolution of these procedures, provided by high field concentrations in sub-wavelength regions near tips, objects or surfaces, involves a large contribution of evanescent waves, namely, of inhomogeneous plane-wave components of both the illuminating and scattered light field. Therefore, in any analysis of the forces exerted by near fields on particles, the role of the evanescent waves is of paramount importance.

One contribution of 13 to a Theme 'Nano-optics and near-field microscopy'. 
The light-intensity enhancements in near-field regions near surfaces may give rise to enhanced gradient forces capable of trapping particles within nanometric scale regions (Novotny et al. 1997; Chaumet et al. 2002a,b). On the other hand, evanescent waves are used in experiments to control the position of a particle suspended over a surface and to estimate the colloidal interaction force between the particle and the surface (Sasaki et al. 1997; Clapp et al. 1999; Dogariu \& Rajagopalan 2000).

This paper presents a review of the main advances in basic studies aimed at understanding the action of optical forces in the near field. After an introduction to the theory of electromagnetic forces in $\S 2$, an illustration of the action of an evanescent wave in a dipolar particle is given in $\S 3$, which provides an interpretative value of the role of the so-called gradient and scattering-plus-absorption force components. Then, the effect of multiple scattering of light on particles over flat substrates is addressed in $\S 4$. This also permits the analysis of the effects of particle nature and size, as well as distance to the substrate, which form the basis for performing controlled experiments. The role of near-field forces upon particles employed as probes for surface-topography sensing is addressed in $\S 5$. Here the role of plasmon-polariton resonances of metallic particles in the signal provided by force enhancements is discussed. Optical binding between illuminated particles is discussed in $\S 6$. Finally, in $\S 7$ we review studies of optical manipulation of nanoparticles with apertureless probes and the interplay of photonic forces and surface-enhanced Raman scattering (SERS), respectively.

\section{Basic theory of forces due to electromagnetic fields}

\section{(a) Maxwell's stress tensor}

The force $\boldsymbol{F}$ on a charge $q$, moving with velocity $\boldsymbol{v}$ in an external electromagnetic field in a medium that can be characterized by a permittivity $\epsilon$ and permeability $\mu$, is given by $\boldsymbol{F}=q(\mathcal{E}+\boldsymbol{v} / c \times \mathcal{B})$, where $\mathcal{E}$ and $\mathcal{B}$ denote the electric field and the magnetic induction vectors, respectively. $\mathcal{B}=\mu \mathcal{H}, \mathcal{H}$ being the magnetic vector. In a system of charges the total force equals the variation $\mathrm{d} \boldsymbol{P}_{\text {mec }} / \mathrm{d} t$ of the mechanical momentum of the system, and it is known (Jackson 1975; Stratton 1941) that one has the conservation law

$$
\frac{\mathrm{d} \boldsymbol{P}_{\text {mec }}}{\mathrm{d} t}+\frac{\mathrm{d} \boldsymbol{P}_{\text {field }}}{\mathrm{d} t}=\int_{S} \boldsymbol{T} \cdot \boldsymbol{n} \mathrm{d} s .
$$

In (2.1) $S$ is any arbitrary closed surface that includes a volume $V$ containing the system of charges; $\boldsymbol{P}_{\text {field }}$ is the total electromagnetic momentum given by Gordon (1973): $\boldsymbol{P}_{\text {field }}=\int_{V} \boldsymbol{S} \mathrm{d} v / c^{2}, c$ denoting the speed of light and $\boldsymbol{S}=c / 4 \pi(\mathcal{E} \times \mathcal{H})$ being the Poynting vector. $\boldsymbol{T}$ is Maxwell's stress tensor, whose components are given by

$$
T_{i j}=\frac{1}{4 \pi}\left[\epsilon \mathcal{E}_{i} \mathcal{E}_{j}+\mu \mathcal{H}_{i} \mathcal{H}_{j}-\frac{1}{2} \delta_{i j}\left(\epsilon \mathcal{E}^{2}+\mu \mathcal{H}^{2}\right)\right], \quad i, j=1,2,3 .
$$

In the common case of an electromagnetic field incident on a finite body, $S$ and $V$ are its surface and volume, respectively, or any surface and volume enclosing it. The electromagnetic vectors in (2.1) and (2.2) correspond to the total field, namely, incident and field re-emitted by the body.

Most experiments are conducted at optical frequencies and thus involve timeaveraged electromagnetic fields. Let those fields be time-harmonic, so that $\mathcal{E}(\boldsymbol{r}, t)=$ 
$\operatorname{Re}\left[\boldsymbol{E}(\boldsymbol{r}) \mathrm{e}^{-\mathrm{i} \omega t}\right]$ and $\mathcal{H}(\boldsymbol{r}, t)=\operatorname{Re}\left[\boldsymbol{H}(\boldsymbol{r}) \mathrm{e}^{-\mathrm{i} \omega t}\right]$ (where Re means the real part). Then, the time average $\langle\cdot\rangle=\lim _{T \rightarrow \infty} 1 / 2 T \int_{-T}^{T}(\cdot) \mathrm{d} t$ in $(2.1)$ yields the mean force:

$$
\langle\boldsymbol{F}\rangle=\left\langle\frac{\mathrm{d} \boldsymbol{P}_{\mathrm{mec}}}{\mathrm{d} t}\right\rangle=\int_{S}\langle\boldsymbol{T}\rangle \cdot \boldsymbol{n} \mathrm{d} s .
$$

In (2.3) one has that $\left\langle\mathrm{d} \boldsymbol{P}_{\text {field }} / \mathrm{d} t\right\rangle=0$, since

$$
\langle\boldsymbol{S}(\boldsymbol{r}, t)\rangle=\frac{c}{8 \pi} \operatorname{Re}\left[\boldsymbol{E}(\boldsymbol{r}) \times \boldsymbol{B}(\boldsymbol{r})^{*}\right]
$$

(Antoci \& Mihich 1998; Chaumet \& Nieto-Vesperinas 2000c; Chaumet 2004), where * denotes the complex conjugate. Also, the time-average of Maxwell's stress tensor is

$$
\left\langle T_{i j}\right\rangle=\frac{1}{8 \pi}\left[\operatorname{Re}\left(E_{i} \epsilon E_{j}^{*}\right)+\operatorname{Re}\left(H_{i} \mu H_{j}^{*}\right)-\frac{1}{2} \delta_{i j}\left(\epsilon|\boldsymbol{E}|^{2}+\mu|\boldsymbol{H}|^{2}\right)\right], \quad i, j=1,2,3 .
$$

Modelling electromagnetic forces therefore involves knowledge of the total field. Several procedures have been used to evaluate these fields in different configurations. The multiple-multipole method has been employed to find the force exerted by a near-infrared illuminated metal tip on a nanometric particle suspended in a liquid (Novotny et al. 1997). The coupled-dipole method was used to calculate the force on one or more particles due to an illuminated flat dielectric surface (Chaumet \& NietoVesperinas $2000 a, b)$ as well as to study the optical binding between the particles (Chaumet \& Nieto-Vesperinas 2001). The finite-difference-time-domain method has also been employed to study these last two phenomena (Okamoto \& Kawata 1999). The integral method has been used to derive the force near a corrugated surface (Lester et al. 2001; Arias-Gonzalez et al. 2002).

\section{(b) The dipole approximation}

Small particles with radius $a \ll \lambda$ respond to an external electromagnetic field with an induced dipole moment $\mathcal{P}$. Therefore, they experience a force (Gordon 1973)

$$
\boldsymbol{F}=(\mathcal{P} \cdot \nabla) \mathcal{E}+\frac{1}{c} \frac{\partial \mathcal{P}}{\partial t} \times \mathcal{B}
$$

Let the external field be time-harmonic. By making use of the relations $\mathcal{P}=$ $\operatorname{Re}\left[\boldsymbol{p}(\boldsymbol{r}) \mathrm{e}^{-\mathrm{i} \omega t}\right], \boldsymbol{B}=(c / \mathrm{i} \omega) \boldsymbol{\nabla} \times \boldsymbol{E}$ and $\boldsymbol{p}=\alpha \boldsymbol{E}, \alpha$ being the particle polarizability, one can write the time-averaged force on the particle as (Chaumet \& Nieto-Vesperinas $2000 c)$

$$
\left\langle F_{j}(\boldsymbol{r})\right\rangle=\frac{1}{2} \operatorname{Re}\left[\alpha E_{k} \frac{\partial E_{k}^{*}(\boldsymbol{r})}{\partial x_{j}}\right], \quad j, k=1,2,3 .
$$

The polarizability of the small particle, including the radiation-reaction term, is

$$
\alpha=\frac{\alpha_{0}}{1-\frac{2}{3} \mathrm{i} k^{3} \alpha_{0}},
$$

where $\alpha_{0}$ is the static polarizability given by the Claussius-Mossotti equation, $\alpha_{0}=$ $a^{3}(\epsilon-1) /(\epsilon+1)$ and $\epsilon=\epsilon_{2} / \epsilon_{0}$ is the ratio of the particle permittivity $\epsilon_{2}$ to that of the surrounding medium $\epsilon_{0}$ (Draine 1988). The wavenumber $k=\sqrt{\epsilon_{0}} k_{0}$, with $k_{0}=\omega / c$. 
For a wave propagating along $\boldsymbol{k}$, the electric-field vector can be written as

$$
\boldsymbol{E}(\boldsymbol{r})=\boldsymbol{E}_{0}(\boldsymbol{r}) \mathrm{e}^{\mathrm{i} \boldsymbol{k} \cdot \boldsymbol{r}} .
$$

Substituting (2.7) into (2.5), one obtains the force experienced by a dipolar particle

$$
\langle\boldsymbol{F}\rangle=\frac{1}{4} \operatorname{Re}[\alpha] \nabla\left|\boldsymbol{E}_{0}\right|^{2}+\frac{1}{2} \boldsymbol{k} \operatorname{Im}[\alpha]\left|\boldsymbol{E}_{0}\right|^{2}-\frac{1}{2} \operatorname{Im}[\alpha] \operatorname{Im}\left[\boldsymbol{E}_{0} \cdot \nabla \boldsymbol{E}_{0}\right],
$$

where Im denotes the imaginary part. The first term is the gradient component of the force, whereas the second term represents the radiation-pressure contribution to the scattering force. In the case of a Rayleigh particle $(k a \ll 1)$, by substituting the above approximation for $\alpha: \alpha=\alpha_{0}+\frac{2}{3} \mathrm{i} k^{3}\left|\alpha_{0}\right|^{2}$, the second contribution can also be expressed as (Van de Hulst 1981)

$$
\left(\frac{|\boldsymbol{E}|^{2}}{8 \pi}\right) C \frac{\boldsymbol{k}}{k}
$$

$C$ being the particle scattering cross-section: $C=\frac{8}{3} \pi k^{4}\left|\alpha_{0}\right|^{2}$. The last term of (2.8) is zero when the field has a single plane-wave component, as in the next case.

\section{Force on a dipolar particle due to an evanescent wave}

Let the small particle be immersed in the electromagnetic field of an evanescent wave, whose electric vector is $\boldsymbol{E}=\boldsymbol{A} \mathrm{e}^{-q z} \mathrm{e}^{\mathrm{i} \boldsymbol{K} \cdot \boldsymbol{R}}$, where $\boldsymbol{r}=(\boldsymbol{R}, z)$ and $\boldsymbol{k}=\left(\boldsymbol{K}, k_{z}\right) ; \boldsymbol{K}$ and $k_{z}$ satisfy $K^{2}+k_{z}^{2}=k^{2}, k^{2}=\omega^{2} \epsilon_{0} / c^{2}$, with $k_{z}=\mathrm{i} q=\mathrm{i} \sqrt{K^{2}-k^{2}}$.

We assume that this field is created by total internal reflection (TIR) at a flat interface between two media of dielectric permittivity ratio $1 / \epsilon$. The incident wave, $\mathrm{s}$ or $\mathrm{p}$ polarized (i.e. with the electric vector either perpendicular or in the plane of incidence), impinges from the denser medium. Without any loss of generality we can choose $\mathrm{O} x z$ as the incidence plane, so that $\boldsymbol{K}=(K, 0)$. Let $t_{\perp}$ and $t_{\|}$be the transmission coefficients for $\mathrm{s}$ and $\mathrm{p}$ polarizations, respectively. The electric vector is

$$
\boldsymbol{E}=(0,1,0) t_{\perp} \mathrm{e}^{\mathrm{i} K x} \mathrm{e}^{-q z},
$$

for s polarization, and

$$
\boldsymbol{E}=(-\mathrm{i} q, 0, K) \frac{t_{\|}}{k} \mathrm{e}^{\mathrm{i} K x} \mathrm{e}^{-q z}
$$

for p polarization.

By introducing the above expressions for the electric vector $\boldsymbol{E}$ into (2.8), we readily obtain the average total force components. The scattering force is contained in the $(x, y)$-plane (that is, the plane containing the propagation wave vector of the evanescent wave), namely:

$$
\left\langle F_{x}\right\rangle=\frac{1}{2}|t|^{2} K \operatorname{Im}[\alpha] \mathrm{e}^{-2 q z},
$$

whereas the gradient-force component, which is directed along $O z$, reads

$$
\left\langle F_{z}\right\rangle=-\frac{1}{2}|t|^{2} q \operatorname{Re}[\alpha] \mathrm{e}^{-2 q z} .
$$

In (3.3) and (3.4) $t$ stands for either $t_{\perp}$ or $t_{\|}$, depending on whether the polarization is $\mathrm{s}$ or $\mathrm{p}$, respectively. 
For an absorbing particle, by using (2.6) for $\alpha$ in (3.3) and (3.4), one gets for the scattering and absorption force

$$
\left\langle F_{x}\right\rangle=\frac{1}{2}|t|^{2} K \mathrm{e}^{-2 q z} \frac{\operatorname{Im}\left[\alpha_{0}\right]+\left(\frac{2}{3}\right) k^{3}\left|\alpha_{0}\right|^{2}}{1+\left(\frac{4}{9}\right) k^{6}\left|\alpha_{0}\right|^{2}},
$$

and for the gradient force

$$
\left\langle F_{z}\right\rangle=-\frac{1}{2}|t|^{2} q \frac{\operatorname{Re}\left[\alpha_{0}\right]}{1+\left(\frac{4}{9}\right) k^{6}\left|\alpha_{0}\right|^{2}} \mathrm{e}^{-2 q z} .
$$

It should be noted that, except for $\operatorname{Re}[\epsilon]$ between -2 and $1, \operatorname{Re}\left[\alpha_{0}\right]$ is positive, thus making the gradient force directed toward the interface. On the other hand, since $\operatorname{Im}\left[\alpha_{0}\right]$ and $\left|\alpha_{0}\right|^{2}$ are always positive, the scattering force (3.5) pushes the particle in the direction of propagation $\boldsymbol{K}$ of the evanescent wave. Of course, these forces increase with decreasing distance from the interface, and are larger for p polarization due to the orientation of the induced polarization in the particle.

In particular, if $k a \ll 1,(3.5)$ becomes

$$
\left\langle F_{x}\right\rangle=\frac{1}{2}|t|^{2} K \mathrm{e}^{-2 q z}\left[a^{3} \operatorname{Im}\left(\frac{\epsilon-1}{\epsilon+2}\right)+\frac{2}{3} k^{3} a^{6}\left|\frac{\epsilon-1}{\epsilon+2}\right|^{2}\right] .
$$

The first term of (3.7) is the radiation pressure of the evanescent wave on the particle due to absorption, whereas the second term corresponds to scattering. This expression can be further condensed as

$$
\left\langle F_{x}\right\rangle=\frac{|t|^{2}}{8 \pi} \frac{K}{k} \mathrm{e}^{-2 q z} C_{\text {ext }},
$$

where the particle extinction cross-section $C_{\text {ext }}$ has been introduced

$$
C_{\text {ext }}=4 \pi k a^{3} \operatorname{Im}\left(\frac{\epsilon-1}{\epsilon+2}\right)+\frac{8}{3} \pi k^{4} a^{6}\left|\frac{\epsilon-1}{\epsilon+2}\right|^{2} .
$$

\section{Forces on particles upon surfaces}

Trapping dielectric particles and micro-organisms was proved to be feasible through the action of gradient-force components, and is at the heart of the optical-tweezer technique (Ashkin et al. 1986). However, it is well established that the predominance of scattering and absorption components, and their repulsive effect on metallic particles which have large extinction coefficients (Ashkin \& Dziedzic 1974), make the trapping of such particles more delicate. Later, gradient-force trapping of sub-wavelength metallic particles was reported (Svoboda et al. 1993).

The first experiments on forces due to evanescent waves, created by TIR at a sapphire-water interface, upon microspheres suspended close to the interface, were reported by Kawata \& Sugiura (1992). Further experiments were done either on a waveguide (Kawata \& Tani 1996), or by attaching the sphere to an atomic force microscope (AFM) cantilever (Vilfan et al. 1998). This work aimed at estimating the magnitude of the force, although they did not conclusively establish its sign, which, as shown in the previous section and in what follows, depends on the particle 


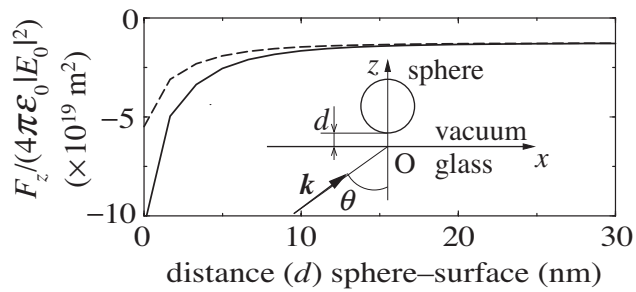

Figure 1. Normalized force in the $z$-direction on a glass sphere with radius $a=10 \mathrm{~nm}$, $\lambda=632.8 \mathrm{~nm}$. The angle of incidence is $\theta=42^{\circ}>\theta_{\mathrm{c}}$. The solid line corresponds to p polarization, and the dashed line to s polarization. The inset is the geometry of the configuration used.

polarizability. Further experiments on the trapping potentials for metallic particles in evanescent fields were conducted (Sasaki et al. 2000), confirming the aforementioned dependence on size and polarizability. Next, we shall analyse this further.

Since the contribution of evanescent waves to fields near surfaces is dominant, it is of interest to study the effect of these components on the force acting on a particle near a flat interface and the effect of multiple scattering on the wave field in which the particle is immersed. Concerning large particles, Mie's scattering theory (Almaas \& Brevick 1995) and ray optics (Walz 1999) have been employed, neglecting the multiple interaction with the substrate. However, further calculations showed that multiple scattering is not at all negligible, and hence it can only be neglected in cases of dielectric particles whose size does not exceed a third of the wavelength and at a distance from the surface no less than the particle radius. Nevertheless, it was shown by Arias-Gonzalez \& Nieto-Vesperinas (2002) that for metallic particles this is only true for the scattering-force component parallel to the surface. However, the oscillations observable in the vertical component of the gradient force as the distance varies are also present at larger separations even in the case of such smaller particles.

\section{(a) Dipolar particles}

The inset in figure 1 shows the geometry used for figures 1 and 2 . Figure 1 shows the $z$-component of the force normalized to $4 \pi \varepsilon_{0}\left|E_{0}\right|^{2}$, where $\left|E_{0}\right|^{2}$ is the intensity of the incident field computed at the position of the particle and $\varepsilon_{0}=8.8542 \times 10^{-12} \mathrm{~F} \mathrm{~m}^{-1}$ is the permittivity of free space. The particle is a small dielectric sphere $(\epsilon=2.25)$, its radius $a=10 \mathrm{~nm}$ and $\lambda=632.8 \mathrm{~nm}$. The angle of incidence is larger than the critical angle $\theta_{c}$. Assuming the sphere to be small, we used (2.6) as well as a selfconsistent method to compute the field and its derivative at the centre of the sphere (Chaumet \& Nieto-Vesperinas 2000a). As the sphere gets closer to the substrate, the normalized force decays significantly. This is due to the interaction of the sphere with the evanescent field scattered by itself and reflected by the substrate at the sphere location. As this field diminishes when $z$ increases, the interaction between the sphere and its own field always produces a negative gradient force. Notice that the decay of the force is stronger for $\mathrm{p}$ polarization, since in that case the $z$ component of the dipole associated with the sphere produces a stronger field than the component parallel to the substrate. When the sphere is far from the substrate, the normalized force becomes constant. As this force is divided by the intensity of the incident field at the particle location, this reflects the fact that the force decreases as $\mathrm{e}^{-2 q z}$ with 

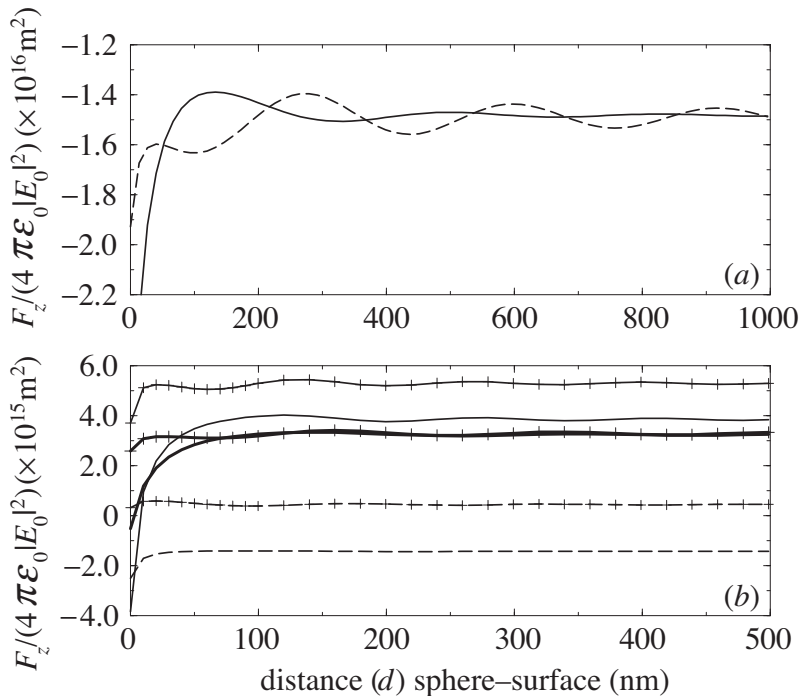

Figure 2. Normalized force in the $z$ direction on a sphere with radius $a=100 \mathrm{~nm}$. (a) Glass sphere with $\lambda=632.8 \mathrm{~nm}, \theta=42^{\circ}>\theta_{\mathrm{c}}$. Solid line, p polarization; dashed line, s polarization. (b) Silver sphere with $\theta=50^{\circ}$ for the following wavelengths: solid line, $\lambda=255 \mathrm{~nm}$; dashed line, $\lambda=300 \mathrm{~nm}$ and; thick solid line, $\lambda=340 \mathrm{~nm}$; symbol + , s polarization; without symbol, $\mathrm{p}$ polarization.

the distance $z$ from the surface (see also (3.3) and (3.4)). This shows that the force only depends on the incident field and there is no interaction between the sphere and the surface.

\section{(b) Particles with sizes of the order of the wavelength}

For larger spheres, the object is represented as a set of dipoles. The self-consistent field at each dipole is computed and used in (2.6) to obtain the force on each element of the discretization (Chaumet \& Nieto-Vesperinas 2000a). Far from the Rayleigh scattering regime $(a=100 \mathrm{~nm})$, figure $2 a$ shows, for a dielectric sphere, that the two polarizations produce oscillations of the $z$ component of the force with period $\lambda / 2$. These oscillations are due to the interaction of the sphere with both the incident field and the propagating waves scattered by the sphere and reflected by the surface. These propagating waves are negligible in the case of a sphere which is small compared with the wavelength. There is also a large difference in the magnitude of these oscillations depending on the polarization. This is a consequence of the different orientations with respect to the substrate of the set of dipoles forming the spheres.

Figure $2 b$ shows the $z$ component of the normalized force on a silver sphere with $a=100 \mathrm{~nm}, \theta=50^{\circ}$. Three wavelengths of illumination $(\lambda=255,300,340 \mathrm{~nm})$ are considered. When the sphere is close to the substrate, we observe, as in the dielectric case, a decay of the force due to the interaction of the sphere with itself. Again, far from the surface, oscillations due to propagating waves appear. However, now the force is always positive, except for p polarization at $\lambda=300 \mathrm{~nm}$. We saw in the previous section (see (3.5)-(3.9)) that for a dipolar particle the $z$ component of the force is due to the gradient force only, whereas the $x$ component is proportional to 


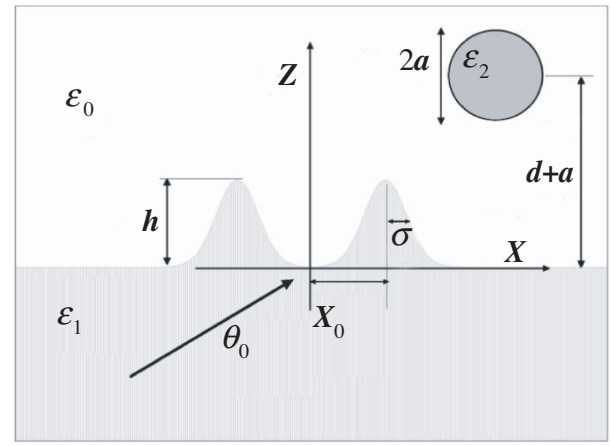

Figure 3. Geometry of the system as discussed in $\S 5$.

the absorption and scattering cross-sections. But for larger particles, the scattering and absorption forces acquire a positive $z$ component due to multiple reflections inside the sphere. Since the absorption and gradient forces are both proportional to $a^{3}$, they have the same order of magnitude. For $\lambda=255 \mathrm{~nm}$ or $340 \mathrm{~nm}$ the real part of the polarizability is negative; hence, both the gradient and the absorption forces are positive (see (3.6)). For $\lambda=300 \mathrm{~nm}$ the real part of the polarizability is positive; thus, there is a negative gradient force and a positive absorption force, which entails different behaviours for the two polarizations. Assuming that the radiative part of the field in the normal direction is larger for s polarization, the absorption force becomes larger than the gradient force. Conversely, for $\mathrm{p}$ polarization the gradient force remains larger than the absorption force, yielding a negative total force.

\section{Forces and surface topography. Nanoparticle resonances}

Field-intensity enhancements due to the excitation of morphology-dependent resonances in small particles (Kreibig \& Vollmer 1995) are well known for both isolated particles and arrays of particles (Maier et al. 2003). Such metallic nanosystems have interesting optical properties. In particular, they can alter radiation pressure (Ashkin \& Dziedzic 1977), and hence they can play a role in near-field photonic forces. They can also be used to enhance the near-field optical signature of confined electromagnetic fields. Gu \& Ke $(1999,2000)$ have demonstrated the use of a laser-trapped metallic particle as a new form of near-field probe. In their experiment the photonic force is used to create a localized probe that will scatter the near field. However, photonic forces can also be used directly to detect topographic variations.

We next discuss the use of particles in transducing surface topography into a force signal. This constitutes a form of near-field photonic force microscopy.

Figure 3 illustrates the geometry for studying the near-field photonic force on a nanometric particle from a surface with defects: a cylinder (the two-dimensional version of a particle) immersed in water $\left(\epsilon_{0}=1.7769\right)$ varies its position over a water-glass interface $\left(\epsilon_{1}=2.3104\right)$. An incident Gaussian beam of half-width at half-maximum $W$, either s or p polarized, is incident from the glass side at angle $\theta_{0}$. We address the electromagnetic force on the nanocylinder illuminated under TIR, so that $\theta_{0}$ is larger than the critical angle $\theta_{\mathrm{c}}=61.28^{\circ}$. Multiple interactions of the scattered wave between the object and the rough interface are considered. Silver cylinders of radius $a$ at distance $d+a$ from the flat portion of the surface 


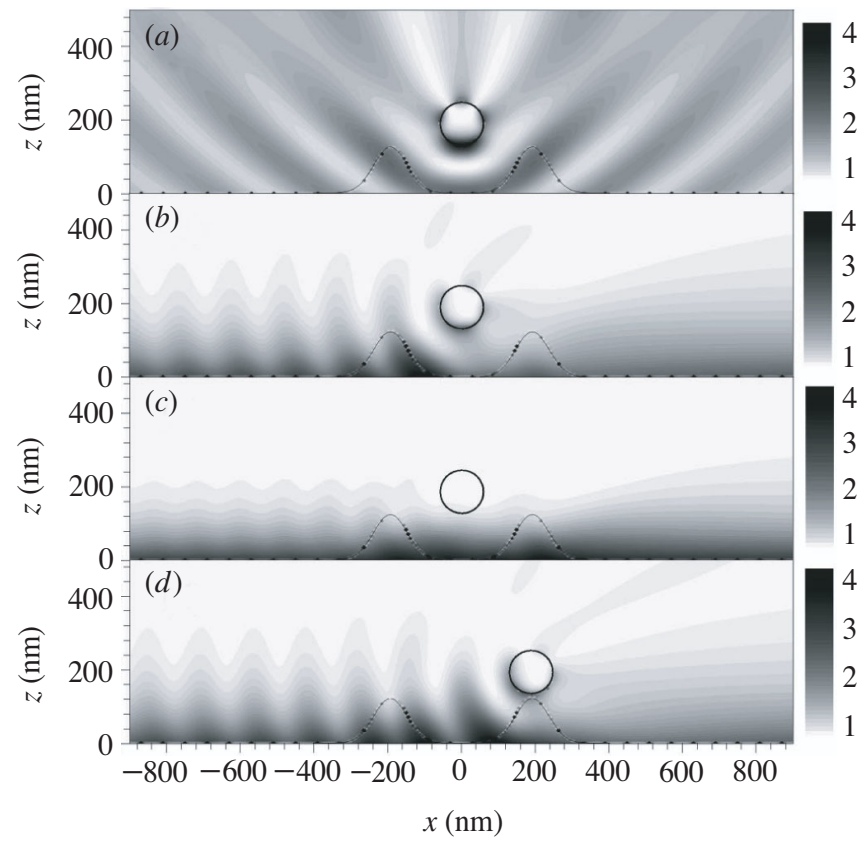

Figure 4. $\left|H / H_{0}\right|^{2}$; p polarization from a silver cylinder with $a=60 \mathrm{~nm}$ immersed in water on a glass substrate with defect parameter $X_{0}=191.4 \mathrm{~nm}, h=127.6 \mathrm{~nm}$ and $\sigma=63.8 \mathrm{~nm}$ at a distance $d=162.6 \mathrm{~nm}$. Gaussian beam incidence with $W=4000 \mathrm{~nm}$. (a) $\lambda=387 \mathrm{~nm}$ (on resonance), $\theta_{0}=0^{\circ}$. (b) $\lambda=387 \mathrm{~nm}$ (on resonance), $\theta_{0}=66^{\circ}$. (c) $\lambda=316 \mathrm{~nm}$ (off resonance), $\theta_{0}=66^{\circ} .(d) \lambda=387 \mathrm{~nm}$ (on resonance), $\theta_{0}=66^{\circ}$. The cylinder is placed at $(0,192.6) \mathrm{nm}$ in $(a)-(c)$, and at $(191.4,192.6) \mathrm{nm}$ in $(d)$.

are addressed. The defects are two protrusions on the flat surface described by the height:

$$
z=h\left[\exp \left(-\frac{\left(x-X_{0}\right)^{2}}{\sigma^{2}}\right)+\exp \left(-\frac{\left(x+X_{0}\right)^{2}}{\sigma^{2}}\right)\right]
$$

For this configuration there is no depolarization in the scattering of either $\mathrm{s}$ or $\mathrm{p}$ waves (Nieto-Vesperinas 1991). The field is rigorously calculated by a self-consistent method, e.g. the extinction-theorem boundary condition (Arias-Gonzalez \& NietoVesperinas 2002). The electromagnetic forces are then obtained from Maxwell's stress tensor (2.4).

The near-field intensity distribution $\left|H / H_{0}\right|^{2}$ corresponding to the configuration of figure 3 is shown in figure 4 (Arias-Gonzalez et al. 2002). A silver cylinder of radius $a=60 \mathrm{~nm}$ varies its position at constant distance $d=162.6 \mathrm{~nm}$ above the interface. The system is illuminated by a p-polarized Gaussian beam ( $W=4000 \mathrm{~nm})$ at $\theta_{0}=0^{\circ}$ and $\lambda=387 \mathrm{~nm}\left(\epsilon_{2}=-3.22+\mathrm{i} 0.70\right)$. Figure $4 a$ shows the aforementioned distribution when the particle is centred between the protrusions. A plasmon resonance is excited, as manifested by the field-intensity enhancement on the cylinder surface. At this resonant wavelength, the main Mie coefficient contributor is $n=2$, which corresponds to the number of lobes $(2 n)$ along this surface (Barber \& Chang 1988). Figure $4 b$ shows the same situation but with $\theta_{0}=66^{\circ}$. The field intensities are markedly different to those of figure $4 c$, in which the wavelength has been changed 

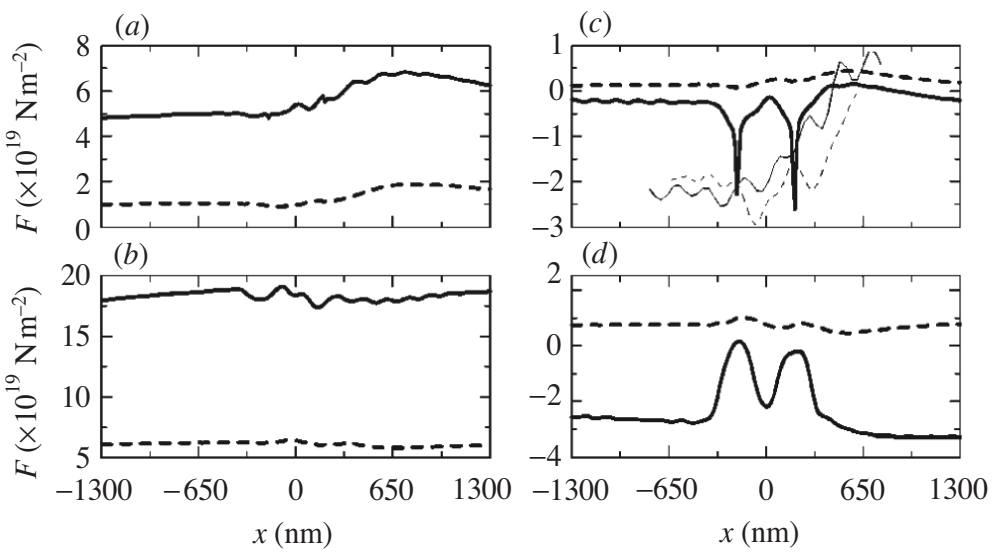

Figure 5. Force on a silver cylinder with $a=60 \mathrm{~nm}$ immersed in water, scanned at constant distance on a glass surface with defect parameters $X_{0}=191.4 \mathrm{~nm}$ and $\sigma=63.8 \mathrm{~nm}$ along $\mathrm{O} x$. The incident field is a p-polarized Gaussian beam with $W=4000 \mathrm{~nm}$ and $\theta_{0}=66^{\circ}$. (a) Horizontal force, $h=127.6 \mathrm{~nm}, d=15 \mathrm{~nm}$. (b) Horizontal force, $h=-127.6 \mathrm{~nm}, d=15 \mathrm{~nm}$. (c) Vertical force, $h=127.6 \mathrm{~nm}, d=162.6 \mathrm{~nm}$. (d) Vertical force, $h=-127.6 \mathrm{~nm}, d=15 \mathrm{~nm}$. Solid curves, $\lambda=387 \mathrm{~nm}$ (on resonance); dashed curves, $\lambda=316 \mathrm{~nm}$ (off resonance). Thin lines in $(c)$ show $\left|H / H_{0}\right|$ (in arbitrary units), averaged on the perimeter of the cylinder cross-section, while it scans the surface. The actual magnitude of the intensity in the resonance case is almost seven times larger than in the non-resonant one.

to $\lambda=316 \mathrm{~nm}\left(\epsilon_{2}=0.78+\mathrm{i} 1.07\right)$ so that there is no particle resonance excitation at all. Figure $4 d$ shows the same as in figure $4 b$ but at a different $x$-position of the particle. Figure $4 b, d$ shows strong perturbations of the intensity map by the presence of the particle. This strong signal makes possible optical force microscopy at resonant conditions with such small metallic particles used as nanoprobes. One can also notice the interference pattern at the left side of the particle, between the evanescent wave and the strongly reflected waves from the cylinder, which in resonant conditions behaves as an efficient radiating antenna (Krenn et al. 1999) due to its much larger scattering cross-section on resonance. The fringe spacing is $\lambda / 2(\lambda$ being the corresponding wavelength in water), and it is the same as that of the fringes below the particle in figure $4 a$.

The variation of the Cartesian components of the electromagnetic force (AriasGonzalez \& Nieto-Vesperinas 2002) are shown in figure $5\left(F_{x}\right.$, figure $5 a, b ; F_{z}$, figure $5 c, d$ ) on displacing the particle at constant distance $d$ above the interface, at either plasmon-resonance excitation $(\lambda=387 \mathrm{~nm}$, solid lines), or off resonance $(\lambda=316 \mathrm{~nm}$, dashed lines). Notice that the resonant wavelength does not change appreciably with the particle position in this system. The incident beam power (per unit length) is $3.9320 \mathrm{~W} \mathrm{~m}^{-1}$, both on resonance and at $\lambda=316 \mathrm{~nm}$. Figure $5 a, c$ shows the force when $h=127.6 \mathrm{~nm}$ (protrusions) and $d=162.6 \mathrm{~nm}$. On the other hand, figure $5 b, d$ displays the force when $h=-127.6 \mathrm{~nm}$ (grooves) and $d=15 \mathrm{~nm}$. The illumination is done with a p-polarized Gaussian beam of $W=4000 \mathrm{~nm}$ at $\theta_{0}=66^{\circ}$. It is seen from these curves that the force distributions resemble the surface topography on resonant conditions with a signal which is remarkably larger than off resonance. This feature is especially manifested in the $z$ component of the force, in which the two protrusions are clearly distinguished from the rest of interference 
ripples, as explained above. Figure $5 c$ also shows (thin lines) the signal that conventional near-field microscopy would measure in this configuration, namely, the normalized magnetic near-field intensity, averaged on the cylinder cross-section. These values are shown in arbitrary units, and in fact the curve corresponding to plasmon resonant conditions is almost seven times larger than that off-resonance. The force distributions also show that resonance excitation also enhances the contrast of the surface-topography image. This has also been observed with other profiles, including surface-relief gratings. Figure $5 b, d$ shows some results for $h$ inverted, namely, the protrusion now being grooves; the vertical component of the force distribution then presents an inverted contrast. Notice that in figure $5 b, d$ the particle is closer to the surface $(d=15 \mathrm{~nm})$ thus giving a higher image contrast. These results show that both the positions and sign of the defect height can be distinguished by the optical force scanning.

In the case of larger particles, multiple scattering with the surface increases, and the presence of a resonance also enhances the intensity around the particle. This, however, yields force signals with less resolution, and whose spatial distribution may present Goos-Hänchen shifts due to evanescent components, and that in some cases may not follow so faithfully the surface topography (Arias-Gonzalez et al. 2002).

\section{Optical binding}

A few years ago Dufresne \& Grier (1998) (see also Dufresne et al. 2001) showed the possibility of creating nanocomposite materials with an array of optical tweezers generated by diffractive optics. More recently, Eriksen et al. (2002) demonstrated the possibility of assembling microstructures with multiple-beam optical tweezers generated by the generalized phase-contrast method (Macdonald et al. 2002). Another way of creating microstructures is to use the interaction between the particles themselves to achieve the assembling. The idea of optical binding was illustrated by Burns et al . (1989) on particles immersed in water illuminated by an intense beam. They observed that the preferred relative separations between the particles related directly to the wavelength of illumination. This effect can be explained from (2.6). We consider two spheres immersed in water under a plane-wave illumination (figure $6 a$ ).

For dipolar spheres, the field at the position of the second sphere is the sum of the incident field and the field scattered by the first sphere

$$
\boldsymbol{E}\left(\boldsymbol{r}_{2}\right)=\boldsymbol{E}_{0}\left(\boldsymbol{r}_{2}\right)+\boldsymbol{S}\left(\boldsymbol{r}_{2}, \boldsymbol{r}_{1}\right) \alpha_{1} \boldsymbol{E}\left(\boldsymbol{r}_{1}\right)
$$

where $\boldsymbol{S}\left(\boldsymbol{r}_{2}, \boldsymbol{r}_{1}\right)$ is free-space field-susceptibility tensor (Jackson 1975). Using (6.1) for the first and second sphere in (2.6) and the fact that the incident wave is a plane wave in the $z$-direction, the $x$ component of the force for the second sphere can be written

$$
F_{x}\left(\boldsymbol{r}_{2}\right)=\frac{1}{2} \operatorname{Re}\left(\alpha_{2} E_{i}\left(\boldsymbol{r}_{2}\right) \alpha_{1}^{*} E_{i}^{*}\left(\boldsymbol{r}_{1}\right) \frac{\partial}{\partial x} S_{i i}^{*}\left(\boldsymbol{r}_{2}, \boldsymbol{r}_{1}\right)\right),
$$

where $i$ stands for $x$ if the polarization of the field is along the $x$-axis and $y$ if the polarization of the field is along the $y$-axis. To get the force on the first sphere the indices ' 1 ' and ' 2 ' must be exchanged. In the case of the experiments of Burns et al. (1989) the particles were identical $\left(\alpha_{1}=\alpha_{2}\right)$; hence the force becomes

$$
F_{x}\left(\boldsymbol{r}_{2}\right)=\frac{1}{2}\left|\alpha_{1} E_{i}\left(\boldsymbol{r}_{1}\right)\right|^{2} \operatorname{Re}\left(\frac{\partial}{\partial x} S_{i i}\left(\boldsymbol{r}_{2}, \boldsymbol{r}_{1}\right)\right) .
$$

Phil. Trans. R. Soc. Lond. A (2004) 
(a)

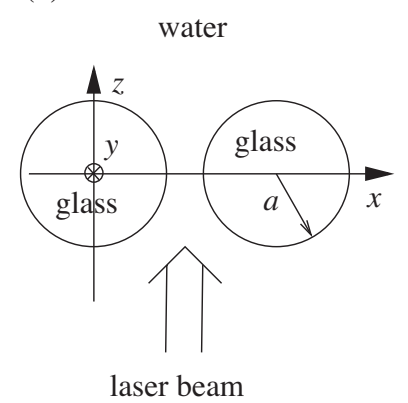

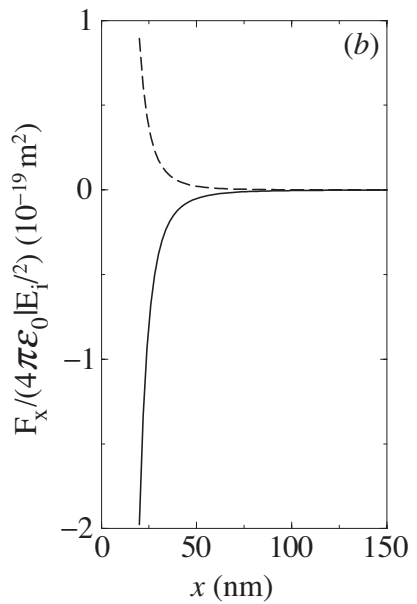

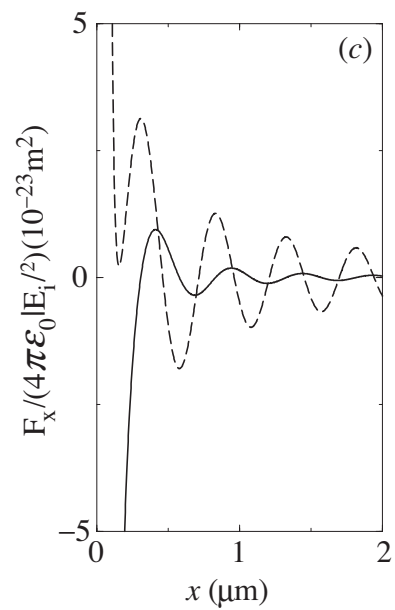

Figure 6. (a) Scheme of the geometry used for studying the optical binding. Both spheres are in glass with $a=10 \mathrm{~nm}$, and $\lambda=632.8 \mathrm{~nm}$, the wavelength in vacuum. (b) Force in near field on the left sphere and $(c)$ force in far field on the left sphere. Solid line is obtained when the polarization of the field is along the $x$-axis; dashed line is obtained when the polarization of the field is along the $y$-axis.

Figure $6 b, c$ shows the optical force in near-field and far-field regimes. To understand the behaviour of the forces, we use (6.3) with some approximations. In near field, i.e. when the distance between the two spheres is small compared with the wavelength, we can make the non-retarded approximation $(k=0)$, which yields, for the derivative of the free-space field-susceptibility tensor,

$$
\begin{array}{cc}
\frac{\partial}{\partial x} S_{i i}\left(\boldsymbol{r}_{2}, \boldsymbol{r}_{1}\right)=\frac{-6\left(x_{2}-x_{1}\right)}{\left|x_{2}-x_{1}\right|^{5}} & \text { if } i=x, \\
\frac{\partial}{\partial x} S_{i i}\left(\boldsymbol{r}_{2}, \boldsymbol{r}_{1}\right)=\frac{3\left(x_{2}-x_{1}\right)}{\left|x_{2}-x_{1}\right|^{5}} & \text { if } i=y .
\end{array}
$$

Under the assumption that $\alpha_{1} S_{i i}\left(\boldsymbol{r}_{2}, \boldsymbol{r}_{1}\right)$ is smaller than one (this approximation assumes that the dipoles associated to the sphere are only induced by the incident field, which is perfectly valid for small glass spheres) we get

$$
\begin{aligned}
& F_{x}\left(\boldsymbol{r}_{2}\right)=\frac{-3\left|\alpha_{1} E_{0_{x}}\right|^{2}}{\left|x_{2}-x_{1}\right|^{4}} \quad \text { for } i=x, \\
& F_{x}\left(\boldsymbol{r}_{2}\right)=\frac{\frac{3}{2}\left|\alpha_{1} E_{0_{y}}\right|^{2}}{\left|x_{2}-x_{1}\right|^{4}} \quad \text { for } i=y .
\end{aligned}
$$

It appears clear that, according to the polarization of the incident field, the spheres either attract $(i=x)$ or repel $(i=y)$ each other. From (6.6) and (6.7) it is easy to explain the repulsive or attractive force. When the field is polarized along $x$, the field due to the first sphere at the location of the second sphere and the dipole associated to the second sphere are in phase. Owing to the gradient force, the second sphere is attracted by the higher-intensity field and goes towards the first sphere. When the polarization of the field is along the $y$-axis the field due to the first sphere at the location of the second sphere and the dipole associated to the second sphere have 


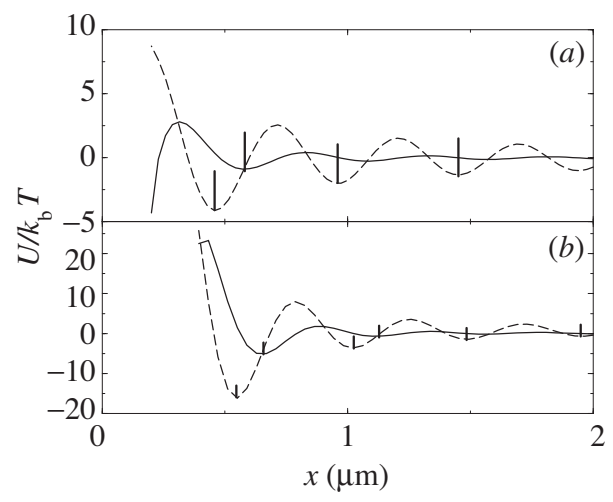

Figure 7. Potential of trapping normalized to $k_{\mathrm{B}} T$ for two identical glass spheres in water. (a) $a=100 \mathrm{~nm}$; (b) $a=200 \mathrm{~nm}$. Solid line, polarization following the $x$-axis; dashed line, polarization following the $y$-axis. The height of the vertical bars corresponds to a normalized potential equal to three.

opposite phases. Hence the second sphere is attracted by the lower intensity field and moves away from the first sphere.

Equation (6.3) in the far field can be written, using the expression of

$$
\left(\frac{\partial}{\partial x}\right) S_{i i}\left(\boldsymbol{r}_{2}, \boldsymbol{r}_{1}\right) \text { for }\left|\boldsymbol{r}_{2}-\boldsymbol{r}_{1}\right|
$$

large compared with the wavelength, as

$$
\begin{array}{ll}
F_{x}\left(\boldsymbol{r}_{2}\right)=\left|\alpha_{1} E_{0_{x}}\right|^{2} \frac{\cos \left(k\left|x_{2}-x_{1}\right|\right)}{\left(x_{2}-x_{1}\right)^{2}} & \text { for } i=x, \\
F_{x}\left(\boldsymbol{r}_{2}\right)=-\left|\alpha_{1} E_{0_{y}}\right|^{2} \frac{\sin \left(k\left|x_{2}-x_{1}\right|\right)}{\left(x_{2}-x_{1}\right)^{2}} & \text { for } i=y .
\end{array}
$$

The previous argument still applies; following the phase relation between the dipole associated to the sphere and the field scattered by the other sphere, the optical force is either attractive or repulsive. This explains the oscillations observed in figure $6 c$. Notice that the oscillations for the two polarizations are shifted by $\lambda / 4$ because they involve cosine and sine terms.

One can also compute the trapping potential normalized to $k_{\mathrm{B}} T$ (with $T=290 \mathrm{~K}$ and $k_{\mathrm{B}}$, the Boltzmann's constant) for a large particle in water. The diameter of the particle is $200 \mathrm{~nm}$ (figure $7 a$ ) and $400 \mathrm{~nm}$ (figure $7 b$ ), and the irradiance of the laser beam is $0.2 \mathrm{~W} \mathrm{\mu m}^{-2}$ and $\lambda=632.8 \mathrm{~nm}$. The trapping potential needs to be larger than the Brownian motion. For instance, we want the potential wells to be deeper than $3 k_{\mathrm{B}} T$. The bars plotted in figure 7 correspond to the $3 k_{\mathrm{B}} T$ limit. We see that when the size of the particle increases, the potential well becomes deeper. The trapping potential is deeper when the polarization is along the $y$-axis and it has a period of one wavelength. These results accurately reproduce the experiments of Burns et al. (1989). Notice that they used larger particles but the irradiance of the laser was weaker. 


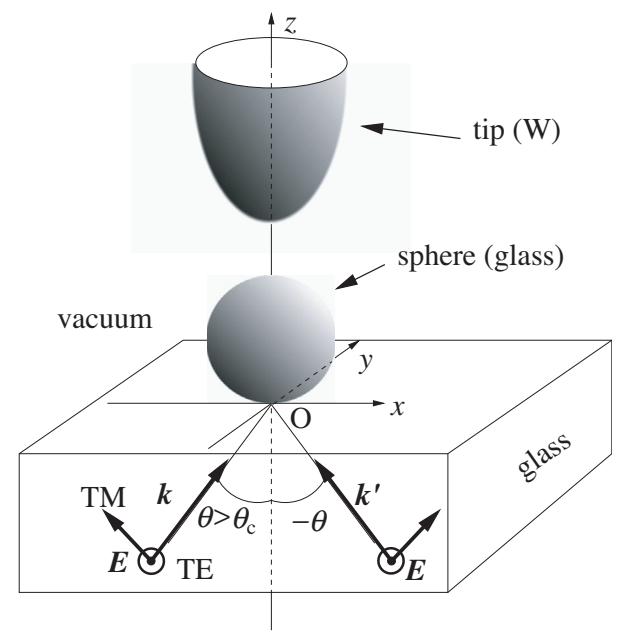

Figure 8. Schematic of the configuration used to manipulate a nanoparticle over a substrate: a dielectric sphere (radius $10 \mathrm{~nm}$ ) on a flat dielectric substrate is illuminated under TIR. A tungsten probe is used to create an optical trap.

\section{Optical tweezers: nanomanipulation with an apertureless probe}

One of the most interesting applications of optical forces is optical tweezers. They have proved useful not only for trapping particles, but also for assembling objects ranging from microspheres to biological cells (Dufresne et al. 2001; Dufresne \& Grier 1998; Macdonald et al. 2002). More recently, optical tweezers have been used to transport Bose-Einstein condensates over large distances (Gustavson et al. 2002). However, most of these manipulations involve objects whose size is of the order of one to several micrometres. It would be interesting to deal with neutral particles of a few nanometres.

One solution consists of using a sharp metallic tip, and the strong enhancement of the field at this metallic tip apex. Novotny et al. (1997) used a gold tip in water illuminated by a monochromatic wave at $\lambda=810 \mathrm{~nm}$; at this wavelength the relative permittivity of gold is large and yields a strong enhancement of the field. The direction of the laser beam was perpendicular to the axis of the tip and the field was polarized along the axis of the tip. Due to the strong discontinuity of the field at the tip apex, one gets a significant enhancement of the field localized at the tip apex. When a particle is in the region of enhancement of the field, the optical force, here the gradient force, attracts the particle towards the tip apex. The particle can be moved by the tip, and then released by turning off the laser illumination.

The technique of Novotny et al. (1997) requires one to find, in water, a particle a few nanometres in size. A possibility would be to wait until a particle falls in the trap, but such an operating mode would not allow for a selective capture. Recently, we have proposed a method for selectively capturing particles (Chaumet et al. 2002a, b). The idea of this nanomanipulation scheme is illustrated in figure 8. A particle with relative permittivity $\varepsilon=2.25$ and radius $a=10 \mathrm{~nm}$ is in either air or vacuum above 


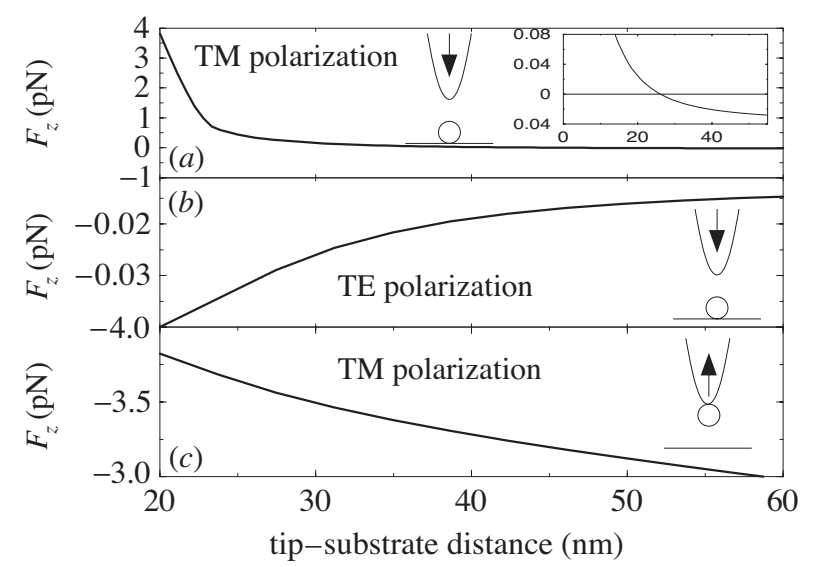

Figure 9. The $z$ component of the force experienced by the sphere versus the distance between the tip and the substrate. We have $a=10 \mathrm{~nm}, \lambda=514.5 \mathrm{~nm}$ and $\theta=43^{\circ}$, and the arrow indicates the direction along which the tip is moved. (a) Situation when the tip approaches the sphere (TM polarization). The inset is an enhancement of $(a)$ near the sign reversal. (b) Situation when the tip approaches the sphere (TE polarization). (c) The tip lifts the sphere in p polarization.

a dielectric substrate. The particle is illuminated by two evanescent waves created by TIR $\left(\theta=43^{\circ}>\theta_{\mathrm{c}}=41.8^{\circ}\right)$ at the substrate-air interface. The two evanescent waves are counterpropagating, i.e. $\boldsymbol{k}_{\|}=-\boldsymbol{k}_{\|}^{\prime}$, with the same polarization and a random phase relation; this is to ensure that the lateral force vanishes. The optical trap is created by the interaction of the incident waves with a tungsten probe with a radius of curvature $r=10 \mathrm{~nm}$ at the apex. The forces are computed for an irradiance of $0.05 \mathrm{~W} \mu \mathrm{m}^{-2}$, which corresponds, for an argon laser $(\lambda=514.5 \mathrm{~nm})$ with a power of $5 \mathrm{~W}$, to a beam focused over an area of $100 \mu \mathrm{m}^{2}$.

Figure $9 a, b$ shows the $z$ component of the force experienced by the sphere versus the tip-substrate distance for both transverse electric (TE) and transverse magnetic (TM) polarization. As the tip gets closer to the sphere one can see that the two polarizations yield different behaviours. First, when the tip is not present (far away from the substrate) one can see that the force is negative. This is due to the fact that the sphere is immersed in the evanescent incident field (which decays with the distance to the substrate), hence the gradient force is negative. For TE polarization, the $z$ component of the force becomes more negative when the distance between the tip and the sphere decreases. Assuming that the sphere and the apex of the tip are two dipoles, it is easy to understand this effect. In TE polarization we have two dipoles, in a first approximation, parallel to the substrate; hence they tend to repel each other, as explained in the previous section. For TM polarization the force becomes positive when the tip gets closer to the sphere. Due to the $z$ component of the field, we obtain the effect described by Novotny et al. (1997), i.e. a large enhancement of the field at the tip apex. Hence, when the tip approaches the sphere, it experiences a positive gradient force which can counterbalance the negative force due to the incident field when the tip is close enough to the particle (inset of figure $9 a$ ). This leads to the trapping of the sphere at the tip apex. It is then possible (figure 9c) to lift the particle off the substrate in TM polarization. Note that the optical force 
decays slowly as the angle of illumination nears the critical value (in TE polarization the force is always negative).

The procedure for selectively manipulating a nanometric particle with an apertureless microscope is as follows. First TE illumination is used, while the tip scans the surface in tapping mode (or in constant-height mode if the area under investigation is small enough). Once an object has been selected, the tip is placed above the object and the polarization of the illumination is rotated to TM. The probe is then brought down over the particle to capture it. Then the tip lifts the particle away from the substrate and moves it to a new position, where it can be released by switching back to a TE polarization. This shows that the lack of trapping capability of the tip under TE illumination is actually an important asset during the imaging/selection, and the release of the particle phases of the manipulation.

Note that while the conventional optical-tweezer technique can be used to trap Mie metallic particles ( $\mathrm{Gu} \&$ Morrish 2002), the scheme described above can be employed to manipulate metallic nanoparticles. Such particles of gold or silver possess unique qualities for creating localized enhancement of electromagnetic fields. One of the most exciting uses of these enhanced electromagnetic near fields is to amplify the weak Raman scattering by molecules for application in biophysics and biochemistry (Kneipp et al. 2002). SERS using metallic nanoparticles can greatly enhance the intrinsic Raman signature of a molecule and has the potential to achieve molecular identification at the single-molecule level.

\section{Conclusion}

Since the first realization that radiation pressure could be used to manipulate matter, the applications of photonic forces have ranged from laser cooling and trapping of atoms and molecules to manipulation and assembling of small particles and biological systems. With the advent of near-field optics and nanophotonics, the possibility of shaping optical fields on the sub-wavelength scale has opened a new realm of applications for photonic forces: a domain where evanescent modes of the electromagnetic fields prevail and where light can be confined to nanometric regions. This paper reviews the basic conceptual and analytical tools needed to address the use of near-field photonic forces for trapping and manipulating small particles. Whereas the use of photonic forces in the near field is still at an early stage, the properties of confined optical fields allied to the advances in far-field photonic forces offer an exciting prospect for the development of new applications in areas such as multiparticle assembling (Dufresne et al. 2001; Eriksen et al. 2002) or micromotors (Collett et al. 2003; Sacconi et al. 2003).

Thanks are given to Ricardo Arias-Gonzalez for work shared through the years. M.N.-V. acknowledges financial support from the Spanish Ministry of Science and Technology.

\section{References}

Almaas, E. \& Brevick, I. 1995 Radiation forces on a micrometer-sized sphere in an evanescent field. J. Opt. Soc. Am. B 12, 2429-2438.

Antoci, S. \& Mihich, L. 1998 Detecting Abraham's force of light by the Fresnel-Fizeau effect. Eur. Phys. J. D 3, 205-210.

Phil. Trans. R. Soc. Lond. A (2004) 
Arias-Gonzalez, J. R. \& Nieto-Vesperinas, M. 2000 Near-field distributions of resonant modes in small dielectric objects on flat surfaces. Opt. Lett. 25, 782-784.

Arias-Gonzalez, J. R. \& Nieto-Vesperinas, M. 2002 Radiation pressure over dielectric and metallic nanocylinders on surfaces: polarization dependence and plasmon-resonance conditions. Opt. Lett. 27, 2149-2151.

Arias-Gonzalez, J. R., Nieto-Vesperinas, M. \& Lester, M. 2002 Modeling photonic force microscopy with metallic particles under plasmon eigenmode excitation. Phys. Rev. B 65, 115402.

Ashkin, A. \& Dziedzic, J. M. 1974 Stability of optical levitation by radiation pressure. Appl. Phys. Lett. 24, 586-588.

Ashkin, A. \& Dziedzic, J. M. 1977 Observation of resonances in the radiation pressure on dielectric spheres. Phys. Rev. Lett. 38, 1351-1355.

Ashkin, A., Dziedzic, J. M., Bjorkholm, J. E. \& Chu, S. 1986 Observation of a single-beam gradient-force optical trap for dielectric particles. Opt. Lett. 11, 288-290.

Barber, P. W. \& Chang, R. K. (eds) 1988 Optical effects associated with small particles. World Scientific.

Burns, M. M., Fournier, J.-M. \& Golovchenko, J. A. 1989 Optical binding. Phys. Rev. Lett. 63, 1233-1236.

Burns, M. M., Fournier, J.-M. \& Golovchenko, J. A. 1990 Optical matter: crystallization and binding in intense optical fields. Science 249, 749-754.

Chaumet, P. C. 2004 Comment on 'Trapping force, force constant and potential depths for dielectric spheres in the presence of spherical aberrations'. Appl. Opt. (In the press.)

Chaumet, P. C. \& Nieto-Vesperinas, M. 2000 a Coupled dipole method determination of the electromagnetic force on a particle over a flat dielectric substrate. Phys. Rev. B 61, 1411914127.

Chaumet, P. C. \& Nieto-Vesperinas, M. $2000 b$ Electromagnetic force on a metallic particle in the presence of a dielectric surface. Phys. Rev. B 62, 11 185-11 191.

Chaumet, P. C. \& Nieto-Vesperinas, M. 2000c Time-averaged total force on a dipolar sphere in an electromagnetic field. Opt. Lett. 25, 1065-1067.

Chaumet, P. C. \& Nieto-Vesperinas, M. 2001 Optical binding of particles with or without the presence of a flat dielectric surface. Phys. Rev. B 64, 035422.

Chaumet, P. C., Rahmani, A. \& Nieto-Vesperinas, M. 2002a Optical trapping and manipulation of nano-object with an apertureless probe. Phys. Rev. Lett. 88, 123601.

Chaumet, P. C., Rahmani, A. \& Nieto-Vesperinas, M. $2002 b$ Selective nanomanipulation using optical forces. Phys. Rev. B 66, 195405.

Clapp, A. R., Ruta, A. G. \& Dickinson, R. B. 1999 Three-dimensional optical trapping and evanescent wave light scattering for direct measurement of long-range forces between a colloidal particle and a surface. Rev. Scient. Instrum. 70, 2627-2636.

Collett, W. L., Ventrice, C. A. \& Mahajan, S. M. 2003 Electromagnetic wave technique to determine radiation torque on micromachines driven by light. Appl. Phys. Lett. 82, 27302732 .

Dogariu, A. C. \& Rajagopalan, R. 2000 Optical traps as force transducers: the effects of focusing the trapping beam through a dielectric interface. Langmuir 16, 2770-2778.

Draine, B. T. 1988 The discrete dipole approximation and its application to interstellar graphite grains. Astrophys. J. 333, 848-872.

Dufresne, E. R. \& Grier, D. G. 1998 Optical tweezers arrays and optical substrates created with diffractive optics. Rev. Scient. Instrum. 69, 1974-1977.

Dufresne, E. R., Spalding, G. C., Dearing, M. T., Sheets, S. A. \& Grier, D. G. 2001 Computergenerated holographic optical tweezer arrays. Rev. Scient. Instrum. 72, 1810-1816.

Eriksen, R. L., Mogensens, P. C. \& Gluckstad, J. 2002 Multiple-beam optical tweezers generated by the generalized phase-contrast method. Opt. Lett. 27, 267-269.

Phil. Trans. R. Soc. Lond. A (2004) 
Gordon, J. P. 1973 Radiation forces and momenta in dielectric media. Phys. Rev. A 8, 14-21.

$\mathrm{Gu}, \mathrm{M} . \&$ Ke, P. 1999 Image enhancement in near-field scanning optical microscopy with lasertrapped metallic particles. Opt. Lett. 24, 74-76.

Gu, M. \& Ke, P. 2000 Depolarization of evanescent waves scattered by laser-trapped gold particles: effect of particle size. J. Appl. Phys. 88, 5415-5420.

Gu, M. \& Morrish, D. 2002 Three-dimensional trapping of Mie metallic particles by the use of obstructed laser beams J. Appl. Phys. 91, 1606-1612.

Gustavson, T. L., Chikkatur, A. P., Leanhardt, A. E., Görlitz, A., Gupta, S., Pritchard, D. E. \& Ketterle, W. 2002 Transport of Bose-Einstein condensates with optical tweezers. Phys. Rev. Lett. 88, 020401-4.

Hotta, J., Sasaki, K., Masuhara, H. \& Morishima, Y. 1998 Laser-controlled assembling of repulsive unimolecular micelles in aqueous solution. J. Phys. Chem. B 102, 7687-7690.

Jackson, J. D. 1975 Classical electrodynamics. Wiley.

Kawata, S. \& Sugiura, T. 1992 Movement of micrometer-sized particles in the evanescent field of a laser-beam. Opt. Lett. 17, 772-774.

Kawata, S. \& Tani, T. 1996 Optically driven Mie particle in an evanescent field along a channeled waveguide. Opt. Lett. 21, 1768-1770.

Kneipp, K., Kneipp, H., Itzkan, I., Dasari, R. R. \& Feld, M. S. 2002 Surface-enhanced Raman scattering and biophysics. J. Phys. Condens. Matter 14, R597-R624.

Kreibig, U. \& Vollmer, M. 1995 Optical properties of metal clusters. Springer.

Krenn, J. R. (and 10 others) 1999 Squeezing the optical near-field zone by plasmon coupling of metallic nanoparticles. Phys. Rev. Lett. 82, 2590-2593.

Lester, M., Arias-Gonzalez, J. R. \& Nieto-Vesperinas, M. 2001 Fundamentals and model of photonic-force microscopy. Opt. Lett. 26, 707-709.

Macdonald, M. P., Paterson, L., Volke-Sepulveda, K., Arlt, J., Sibbet, W. \& Dholakia, K. 2002 Creation and manipulation of three-dimensional optically trapped structures. Science $\mathbf{2 9 6}$, 1101-1103.

Maier, S. A., Kik, P. G., Atwater, H. A., Meltzer, S., Harel, E., Koel, B. E. \& Requicha, A. A. G. 2003 Local detection of electromagnetic energy transport below the diffraction limit in metal nanoparticle plasmon waveguides. Nature Mater. 2, 229-232.

Misawa, H., Sasaki, K., Koshioka, M., Kitamura, N. \& Masuhara, H. 1992 Multibeam laser manipulation and fixation of microparticles. Appl. Phys. Lett. 60, 310-312.

Nieto-Vesperinas, M. 1991 Scattering and diffraction in physical optics, ch. 7. Wiley.

Novotny, L., Bian, R. X. \& Xie, X. S. 1997 Theory of nanometric optical tweezers. Phys. Rev. Lett. 79, 645-648.

Okamoto, K. \& Kawata, S. 1999 Radiation force exerted on sub-wavelength near a nanoaperture. Phys. Rev. Lett. 83, 4534-4537.

Pralle, A., Florin, E. L., Stelzer, E. H. K. \& Horber, J. K. H. 1998 Local viscosity probed by photonic force microscopy, Appl. Phys. A 66, S71-S73.

Sacconi, L., Romano, G., Ballerini, R., Capitano, M., De Pas, M., Giuntini, M., Dunlap, D., Finzi, L. \& Pavone, F. S. 2003 Three-dimensional magneto-optic trap for micro-object manipulation. Opt. Lett. 26, 1359-1361.

Sasaki, K., Koshioka, M., Misawa, H., Kitamura, N. \& Masuhara, H. 1991 Pattern formation and flow control of fine particles by laser scanning micromanipulation. Opt. Lett. 11, 288-290.

Sasaki, K., Tsukima, M. \& Masuhara, H. 1997 Three-dimensional potential analysis of radiation pressure exerted on a single microparticle. Appl. Phys. Lett. 71, 37-39.

Sasaki, K., Hotta, J., Wada, K. \& Masuhara, H. 2000 Analysis of radiation pressure exerted on a metallic particle within an evanescent field. Opt. Lett. 25, 1385-1387.

Smith, S. B., Cui, Y. \& Bustamante, C. 1996 Overstretching B-DNA: the elastic response of individual double-stranded and single-stranded DNA molecules. Science 271, 795-799.

Phil. Trans. R. Soc. Lond. A (2004) 
Stratton, J. A. 1941 Electromagnetic theory. New York: McGraw-Hill.

Svoboda, K., Schmidt, C. F., Schnapp, B. J. \& Block, S. M. 1993 Direct observation of kinesin stepping by optical trapping interferometry. Nature 365, 721-727.

Van de Hulst, H. C. 1981 Light scattering by small particles. New York: Dover.

Vilfan, M., Musevic, I. \& Copic, M. 1998 AFM observation of force on a dielectric sphere in the evanescent field of totally reflected light. Europhys. Lett. 43, 41-46.

Walz, J. Y. 1999 Ray optics calculation of the radiation forces exerted on a dielectric sphere in an evanescent field. Appl. Opt. 38, 5319-5330. 